\title{
Levantamento fitossociológico das Comunidades de Plantas Infestantes em Áreas de Produção de ARRoz IRRIGado Cultivado SOB Diferentes Sistemas de MANEJO ${ }^{1}$
}

\author{
Phyto-sociological Survey of Weed Communities in Flooded Rice Areas Cultivated Under \\ Different Management Systems
}

ERASMO, E.A.L. ${ }^{2}$, PINHEIRO, L.L.A. ${ }^{3}$ e COSTA, N.V. ${ }^{4}$

\begin{abstract}
RESUMO - O presente trabalho de pesquisa teve como objetivo identificar a composição florística de comunidades de plantas daninhas presentes em áreas agrícolas de várzea, manejadas em diferentes sistemas. O trabalho foi desenvolvido em áreas de produção de arroz irrigado das cooperativas: Cooperativas Mista Rural Vale do Javaés e Cooperativa Agroindustrial Rio Formoso, em Formoso do Araguaia-TO. Foram separadas três áreas de 1 ha, sendo: 1 - área sem rotação de culturas (arroz/pousio) há mais de cinco anos; 2 - área com rotação de culturas (arroz/soja) há mais de cinco anos; 3 - área com rotação de culturas (arroz/melancia) há mais de dois anos. Para caracterização e estudo fitossociológico da comunidade infestante foi utilizado, como unidade amostral, um quadro (1,0 x 1,0 m), lançado aleatoriamente dentro da área de estudo (método do quadrado inventário), por meio de um caminhamento em ziguezague. $\mathrm{Na}$ área sem rotação, foram identificadas 8 famílias e 16 espécies, destacando-se a família Poaceae com maior número espécies; Fimbristylis miliacea (Cyperaceae) foi a espécie com o maior índice de importância relativa $(84,46 \%)$. Na área com rotação arroz/soja, foram identificadas 8 famílias e 12 espécies, destacando-se as famílias Poaceae e Cyperaceae com maior número espécies; Cyperus esculentus (Cyperaceae) foi a espécie com o maior índice de importância relativa $(91,4 \%)$. Na área com rotação arroz/ melancia foram identificadas seis famílias e oito espécies, destacando-se as famílias Euphorbiaceae e Lamiaceae com maior número espécies; Physalis angulata (Solanaceae) foi a espécie com o maior índice de importância relativa $(153,1 \%)$, seguida por Eclipta alba (Compositae) e Hyptis lophanta (Lamiaceae), com 40,45 e 37,6\%, respectivamente.
\end{abstract}

Palavras-chave: várzea, rotação de culturas, estudo fitossociológico, plantas daninhas.

\begin{abstract}
This research aimed to identify the floristic composition of weed communities present in lowland agricultural areas under different management systems. The trial was carried out in flooded rice areas of two different co-ops located in Formoso do Araguaia, Tocantins, Brazil: Mista Rural Vale do Javaés Co-op and Agroindustrial Rio Formoso Co-op. Three different 1 ha areas were selected: 1 - area under 5- year no crop rotation system (rice/fallow); 2 - area under 5- year rice/soybean crop rotation system; and 3 - area under 2-year rice/water-melon crop rotation system. The characterization and phyto-sociological studies of the weed community were carried out using square samples of $1.0 \times 1.0$ meter randomly placed inside each selected area (Inventory Square Method). Eight families and 16 species were identified in the no-crop rotation area, with the family Poaceae presenting the largest number of species and Fimbristylis miliacea (Cyperaceae) presenting the highest relative importance value (84.46\%). The 5-year rice soybean crop rotation system area had 8 families and 12 species with the Poaceae and Cyperaceae presenting the largest number of species and Cyperus esculentus (Cyperaceae) the highest relative importance
\end{abstract}

Recebido para publicação em 1.9.2003 e na forma revisada em 18.6.2004.

2 Eng.-Agr., Dr., Professor da Fundação Universidade do Tocantins/UNITINS, Campus de Gurupi, Caixa Postal 66, 77400-000, Gurupi-TO <erasmolemus @ uol.com.br>. ${ }^{3}$ Eng.-Agr. da Fundação Universidade do Tocantins - UNITINS, Campus de Gurupi, bolsista/CNPq. ${ }^{4}$ Eng.-Agr., aluno do Curso de Pós-Graduação da Universidade Estadual Paulista - FCA/UNESP, Caixa Postal 237, 18603-970 Botucatu-SP, <neumarciovc@bol.com.br>, bolsista/CNPq.

Planta Daninha, Viçosa-MG, v.22, n.2, p.195-201, 2004 
value (91. $40 \%$ ). Six families and 8 species were identified in the 2-year rice/water-melon crop rotation system area, with the Euphorbiaceae and Lamiaceae families presenting the largest number of species. Physalis angulata (Solanaceae) showed the highest relative importance value (153.10\%), followed by Eclipta alba (Compositae) and Hyptis lophanta (Lamiaceae) with a relative importance value of $40.50 \%$ and $37.60 \%$, respectively.

Key words: lowlands, crop rotation, phyto-sociological study, weed.

\section{INTRODUÇÃO}

O Estado do Tocantins caracteriza-se por uma diversidade de ecossistemas, com áreas consideradas aptas para lavouras em torno de $36.000 \mathrm{~km}^{2}$, em que se destacam aquelas denominadas áreas de várzea, com 80.000 ha de arroz irrigado (Jica, 1998).

Essas áreas, por apresentarem normalmente boa fertilidade e umidade, favorecem o surgimento de algumas espécies denominadas plantas daninhas, as quais competem pelos mesmos fatores de crescimento das culturas, acarretando perdas na produção e qualidade de grãos, motivo pelo qual são alvos de controle.

Dentre os diferentes sistemas de controle adotados, a utilização de herbicidas destacase, em razão da sua maior eficiência e facilidade, porém o seu sucesso depende de uma série de princípios técnicos. A identificação das espécies daninhas a serem controladas constitui-se em um desses princípios, visto que a escolha do ingrediente ativo do produto a ser utilizado dependerá do tipo de planta daninha existente no local, além da cultura plantada.

Uma vez que as comunidades infestantes podem variar sua composição florística em função do tipo e da intensidade de tratos culturais impostos, o reconhecimento das espécies presentes torna-se fundamental, quanto mais se for levado em conta o custo financeiro e ambiental da utilização de produtos químicos. Dessa maneira, é importante investir em métodos que auxiliem no conhecimento dessas comunidades.

Um dos métodos mais utilizados no reconhecimento florístico em áreas agrícolas ou não é o denominado estudo fitossociológico, que pode ser conceituado como "a ecologia da comunidade vegetal e envolve as inter-relações de espécies vegetais no espaço e, de certo modo, no tempo" (Martins, 1985). A aplicação de um método fitossociológico ou quantitativo num dado local e num dado tempo permite fazer uma avaliação momentânea da composição da vegetação, obtendo dados de freqüência, densidade, abundância, índice de importância relativa e coeficiente de similaridade das espécies ocorrentes naquela formação. Assim, o método fitossociológico é uma ferramenta que, se usada adequadamente, permite fazer várias inferências sobre a comunidade em questão.

Com base no exposto anteriormente, foi elaborado o presente trabalho de pesquisa, que teve como objetivo identificar a composição florística de comunidades de plantas daninhas presentes em áreas agrícolas de várzea na região de Formoso do Araguaia-TO, manejadas em diferentes sistemas de produção de arroz irrigado.

\section{MATERIAL E MÉTODOS}

Esta pesquisa foi desenvolvida em áreas de produção de arroz irrigado das cooperativas: Cooperativas Mista Rural Vale do Javaés e Cooperativa Agroindustrial Rio Formoso, localizadas na região de Formoso do AraguaiaTO, a $11^{\circ} 43^{\prime} 45^{\prime \prime} \mathrm{S}, 4^{\circ} 04^{\prime} 07^{\prime \prime} \mathrm{N}$ e $280 \mathrm{~m}$ de altitude. Foram separadas três áreas de 1 ha, conforme os métodos de manejo utilizados: área sem rotação de culturas (arroz/pousio) há mais de cinco anos; área com rotação de culturas (arroz/soja) há mais de cinco anos; e área com rotação de culturas (arroz/melancia) há mais de dois anos.

Para caracterização e estudo fitossociológico da comunidade infestante foi utilizado como unidade amostral um quadro $(1,0 \mathrm{x}$ $1,0 \mathrm{~m})$, lançando-se 10 quadros aleatoriamente dentro de cada área de estudo (método do quadrado inventário), por meio de um 
caminhamento em ziguezague. O período de amostragem foi realizado antes do plantio da cultura do arroz irrigado. Em cada quadro amostrado as plantas foram identificadas segundo a família, o gênero e a espécie, bem como foi feita a determinação do número presente de cada espécie. A partir da contagem das espécies presentes, foram calculados os seguintes parâmetros fitossociológicos: densidade (D) e densidade relativa (Dr), calculados segundo a fórmula proposta por Curtis \& Mcintosh (1950); freqüência $(\mathrm{F})$, conforme a fórmula proposta por Martins (1978); freqüência relativa $(\mathrm{Fr})$, abundância relativa (Ar) e indice de importância relativa (Ir), de acordo com a fórmula proposta por Mueller-Dombois \& Ellenberg (1974); abundância (A), segundo a fórmula proposta por Braun-Blanquet (1979); e coeficiente de similaridade, baseando-se na fórmula proposta por Sorensen (1972).

\section{RESULTADOS E DISCUSSÃO}

Na área de produção de arroz irrigado sem rotação de culturas há mais de cinco anos (Tabelas 1 e 2), foram identificadas 8 famílias e 16 espécies, destacando-se a família Poaceae com cinco espécies. As espécies que apresentaram as maiores freqüências foram Cyperus iria, Hyptis crenata, Ludwigia sp. e Eleusine indica, com valores na ordem de 1,0 , seguidas por Murdannia nudiflora com 0,8 e Fimbristylis miliacea com 0,9; estas espécies também apresentaram os maiores valores de freqüência relativa. As espécies com maior densidade relativa foram Cyperus iria, Murdannia nudiflora e Frimbristylis miliacea, com 24,4, 27,6 e $38,8 \%$, respectivamente. Fimbristylis miliacea $(34,95 \%)$, Murdannia nudiflora $(27,95 \%)$ e Cyperus iria $(19,81 \%)$ foram as espécies que apresentaram os maiores valores de abundância relativa, bem como os maiores índices de importância relativa, com valores de 84,46, 65,12 e $56,15 \%$, respectivamente.

$\mathrm{Na}$ área de produção de arroz irrigado rotacionada com a cultura da soja há mais de cinco anos (Tabelas 3 e 4), foram identificadas 8 famílias e 12 espécies, destacando-se as famílias Poaceae e Cyperaceae, com o maior número de espécies. As espécies que apresentaram as maiores freqüências foram Digitaria horizontalis e Echinochloa colonum, com valores na ordem de 1,0 e 0,8 , respectivamente. No entanto, a espécie que apresentou densidade relativa superior à das demais foi Cyperus esculentus, com 40,65\%, seguida por Digitaria horizontalis com 22,12\% e Echinochloa colonum com 21,26\%. Cyperus esculentus, Digitaria horizontalis e Echinochloa colonum foram as espécies que apresentaram os maiores índices de importância relativa, com valores na ordem de $91,4,57,75$ e $55,15 \%$, respectivamente.

$\mathrm{Na}$ área de produção de arroz irrigado rotacionada com a cultura da melancia há mais de dois anos (Tabelas 5 e 6), foram identificadas seis famílias e oito espécies, destacando-se as famílias Euphorbiaceae e Lamiaceae, com o maior número de espécies. Physalis angulata, Eclipta alba e Chamaesyce prostata apresentaram os maiores valores de freqüência, na ordem de 1,0 para cada espécie, seguidas por Hyptis crenata, com 0,7. Contudo, Physalis angulata foi a espécie que obteve os maiores valores de densidade e abundância relativa, na ordem de 72,7 e $60,4 \%$, respectivamente.

Hyptis lophanta, Eclipta alba e Physalis angulata foram as espécies que apresentaram os maiores indices de importância relativa, com valores de $37,60,40,45$ e $153,10 \%$, respectivamente.

Tabela 1 - Distribuição das plantas daninhas por família e espécie, coletadas em área de produção de arroz irrigado sem rotação de cultura há mais de cinco anos. Formoso do Araguaia-TO - 2000

\begin{tabular}{|c|c|c|}
\hline \multirow{2}{*}{ Família } & \multicolumn{2}{|c|}{ Espécie } \\
\hline & Nome científico & Nome comum \\
\hline \multirow{5}{*}{ Poaceae } & Brachiaria plantaginea & Capim-marmelada \\
\hline & Digitaria ciliaris & Digitária \\
\hline & Digitaria horizontalis & Capim-colchão \\
\hline & Echinochloa colonum & Capim-arroz \\
\hline & Eleusine indica & Capim-pé-de-galinha \\
\hline Compositae & $\begin{array}{l}\text { Ageratum conyzoides } \\
\text { Eclipta alba } \\
\text { Emilia sonchifolia }\end{array}$ & $\begin{array}{l}\text { Mentrasto } \\
\text { Erva-de-botão } \\
\text { Falsa-serralha } \\
\end{array}$ \\
\hline Commelinaceae & $\begin{array}{l}\text { Commelina benghalensis } \\
\text { Murdannia nudiflora }\end{array}$ & $\begin{array}{l}\text { Trapoera } \\
\text { Trapoerabinha }\end{array}$ \\
\hline Cyperaceae & $\begin{array}{l}\text { Cyperus iria } \\
\text { Fimbristylis miliacea }\end{array}$ & $\begin{array}{l}\text { Junquinho } \\
\text { Pelunco }\end{array}$ \\
\hline Convolvulaceae & Ipomoea grandifolia & Corda-de-viola \\
\hline Euphorbiaceae & Chamaesyce hirta & Erva-de-santa-luzia \\
\hline Lamiaceae & Hyptis crenata & Cheirosa \\
\hline Onagraceae & Ludwigia sp. & Cruz-de-malta \\
\hline
\end{tabular}


Tabela 2 - Valores de freqüência (F), frequiência relativa (Fr), densidade (D), densidade relativa (Dr), abundância (A), abundância relativa (Ar) e importância relativa (Ir), numa comunidade de plantas daninhas presente em áreas de produção de arroz sem rotação de culturas há mais de cinco anos. Formoso do Araguaia-TO - 2000

\begin{tabular}{|c|c|c|c|c|c|c|c|c|c|}
\hline Espécie & $\begin{array}{l}\text { Quadro } \\
\text { Ocupado }\end{array}$ & $\begin{array}{l}\text { Número de } \\
\text { indivíduos }\end{array}$ & $\mathrm{F}$ & $\begin{array}{l}\text { Fr } \\
(\%)\end{array}$ & 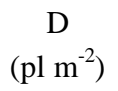 & $\begin{array}{l}\mathrm{Dr} \\
(\%)\end{array}$ & A & $\begin{array}{l}\mathrm{Ar} \\
(\%)\end{array}$ & $\begin{array}{c}\mathrm{Ir} \\
(\%)\end{array}$ \\
\hline Fimbristylis miliacea & 9 & 4806 & 0,90 & 10,71 & 480,60 & 38,80 & 534,00 & 34,90 & 84,46 \\
\hline Murdannia nudiflora & 8 & 3421 & 0,80 & 9,52 & 342,10 & 27,60 & 427,63 & 27,90 & 65,12 \\
\hline Cyperus iria & 10 & 3027 & 1,00 & 11,90 & 302,70 & 24,40 & 302,70 & 19,80 & 56,15 \\
\hline Digitaria ciliaris & 5 & 337 & 0,50 & 5,95 & 33,70 & 2,72 & 67,40 & 4,41 & 13,08 \\
\hline Hyptis crenata & 10 & 186 & 1,00 & 11,90 & 18,60 & 1,50 & 18,60 & 1,22 & 14,62 \\
\hline Ludwigia sp. & 10 & 151 & 1,00 & 11,90 & 15,10 & 1,22 & 15,10 & 0,99 & 14,11 \\
\hline Echinochloa colonum & 2 & 146 & 0,20 & 2,38 & 14,60 & 1,18 & 73,00 & 4,78 & 8,34 \\
\hline Eleusine indica & 10 & 128 & 1,00 & 11,90 & 12,80 & 1,03 & 12,80 & 0,84 & 13,77 \\
\hline Brachiaria plantaginea & 3 & 68 & 0,30 & 3,57 & 6,80 & 0,55 & 22,67 & 1,48 & 5,60 \\
\hline Eclipta alba & 5 & 42 & 0,50 & 5,95 & 4,20 & 0,34 & 8,40 & 0,55 & 6,84 \\
\hline Digitaria horizontalis & 1 & 24 & 0,10 & 1,19 & 2,40 & 0,19 & 24,00 & 1,57 & 2,95 \\
\hline Chamaesyce hirta & 2 & 27 & 0,20 & 2,38 & 2,70 & 0,22 & 13,50 & 0,88 & 3,48 \\
\hline Ipomoea grandifolia & 4 & 12 & 0,40 & 4,76 & 1,20 & 0,10 & 3,00 & 0,20 & 5,06 \\
\hline Commelina benghalensis & 3 & 10 & 0,30 & 3,57 & 1,00 & 0,08 & 3,33 & 0,22 & 3,87 \\
\hline Emilia sonchifolia & 1 & 1 & 0,10 & 1,19 & 0,10 & 0,01 & 1,00 & 0,07 & 1,27 \\
\hline Ageratum conyzoides & 1 & 1 & 0,10 & 1,19 & 0,10 & 0,01 & 1,00 & 0,07 & 1,27 \\
\hline Total & - & $12.387,0$ & 8,40 & 100,00 & $1.238,7$ & 100,00 & $1.528,1$ & 100,00 & 986,80 \\
\hline
\end{tabular}

Tabela 3 - Distribuição das plantas daninhas por família e espécie, coletadas em área de rotação arroz irrigado/ soja há mais de cinco anos. Formoso do Araguaia-TO 2000

\begin{tabular}{|l|l|l|}
\hline \multirow{2}{*}{ Família } & \multicolumn{2}{|c|}{ Espécie } \\
\cline { 2 - 3 } & \multicolumn{1}{|c|}{ Nome científico } & \multicolumn{1}{c|}{ Nome comum } \\
\hline \multirow{3}{*}{ Poaceae } & Digitaria horizontalis & Capim-colchão \\
\cline { 2 - 3 } & Echinochloa colonum & Capim-arroz \\
\cline { 2 - 3 } & Setaria geniculata & Capim-rabo-de-raposa \\
\hline \multirow{2}{*}{ Cyperaceae } & Cyperus iria & Junquinho \\
\cline { 2 - 3 } & Fimbristylis miliacea & Pelunco \\
\hline Compositae & Eclipta alba & Erva-de-botão \\
\hline Euphorbiaceae & Chamaesyce hyssopifolia & Erva-de-andorinha \\
\hline Fabaceae & Senna obtusifolia & Fedegoso-branco \\
\hline Malvaceae & Malachra fasciata & Malva-brava \\
\hline Onagraceae & Ludwigia sp. & Cruz-de-malta \\
\hline Portulacaceae & Portulaca oleracea & Beldroega \\
\hline
\end{tabular}

$\mathrm{Na}$ análise do quociente de similaridade nos diferentes sistemas de produção de arroz irrigado, constataram-se valores de $75 \%$ entre as áreas com sistema de produção de arroz/ sem rotação e na área com sistema de produção com rotação arroz/soja há mais de cinco anos. Quando comparadas as áreas de produção de arroz irrigado sem rotação e a área com rotação arroz/melancia há mais de dois anos, obteve-se um quociente de $22 \%$. No caso das áreas de sistema de produção com rotação arroz/soja há mais de cinco anos e da área com rotação arroz/melancia há mais de dois anos, o valor do quociente correspondeu a $66 \%$.

Através do estudo realizado, verificou-se grande diversidade de espécies presentes nas diferentes áreas estudadas, o que pode ser explicado pelas diferenças entre os solos, pelos tipos de herbicida e pela adubação aplicada segundo as rotações de cultura utilizadas. No entanto, muitas espécies foram constantes na sua presença em todas as áreas, a exemplo de Eclipta alba e Ludwigia sp., podendo isso ser explicado pela adaptação dessas espécies a ambientes de elevada umidade, característica de solos de várzeas.

Houve maior predominância de espécies monocotiledôneas nas áreas de produção de arroz irrigado sem rotação e na rotacionada com soja, apesar da utilização pelas cooperativas de herbicidas registrados com diferentes 
Tabela 4 - Valores de frequiência (F), freqüência relativa (Fr), densidade (D), densidade relativa (Dr), abundância (A), abundância relativa (Ar) e importância relativa (Ir), numa comunidade de plantas daninhas presente em áreas de produção de arroz com rotação de culturas (arroz/soja) há mais de cinco anos. Formoso do Araguaia-TO - 2000

\begin{tabular}{|c|c|c|c|c|c|c|c|c|c|}
\hline Espécie & $\begin{array}{c}\text { Quadro } \\
\text { ocupado }\end{array}$ & $\begin{array}{l}\text { Número de } \\
\text { indivíduos }\end{array}$ & $\mathrm{F}$ & $\begin{array}{c}\mathrm{Fr} \\
(\%)\end{array}$ & $\begin{array}{c}\mathrm{D} \\
\left(\mathrm{pl} \mathrm{m}^{-2}\right)\end{array}$ & $\begin{array}{c}\mathrm{Dr} \\
(\%)\end{array}$ & A & $\begin{array}{c}\mathrm{Ar} \\
(\%) \\
\end{array}$ & $\begin{array}{c}\mathrm{Ir} \\
(\%)\end{array}$ \\
\hline Cyperus esculentus & 7 & 522 & 0,70 & 15,50 & 52,20 & 40,65 & 74,50 & 35,20 & 91,40 \\
\hline Digitaria horizontalis & 10 & 284 & 1,00 & 22,20 & 28,40 & 22,12 & 28,40 & 13,41 & 57,75 \\
\hline Echinochloa colonum & 8 & 273 & 0,80 & 17,70 & 27,30 & 21,26 & 34,10 & 16,11 & 55,15 \\
\hline Cyperus iria & 3 & 100 & 0,30 & 6,67 & 10,00 & 7,79 & 33,30 & 15,73 & 30,19 \\
\hline Eclipta alba & 3 & 47 & 0,30 & 6,67 & 4,70 & 3,66 & 15,60 & 7,40 & 17,72 \\
\hline Setaria geniculata & 4 & 25 & 0,40 & 8,89 & 2,50 & 1,95 & 6,25 & 2,95 & 13,79 \\
\hline Chamaesyce hyssopifolia & 3 & 12 & 0,30 & 6,67 & 1,20 & 0,93 & 4,00 & 1,89 & 9,49 \\
\hline Portulaca oleracea & 2 & 5 & 0,20 & 4,44 & 0,50 & 0,39 & 2,50 & 1,18 & 6,01 \\
\hline Senna obtusifolia & 2 & 6 & 0,20 & 4,44 & 0,60 & 0,74 & 3,00 & 1,42 & 6,33 \\
\hline Ludwigia sp. & 1 & 4 & 0,10 & 2,22 & 0,40 & 0,31 & 4,00 & 1,89 & 4,42 \\
\hline Fimbristylis miliacea & 1 & 4 & 0,10 & 2,22 & 0,40 & 0,31 & 4,00 & 1,89 & 4,42 \\
\hline Malachra fasciata & 1 & 2 & 0,10 & 2,22 & 0,20 & 0,16 & 2,00 & 0,94 & 3,32 \\
\hline Total & - & 1.284 & 4,50 & 100,00 & $128,4,00$ & 100,00 & 212,00 & 100,00 & 300,00 \\
\hline
\end{tabular}

Tabela 5 - Distribuição das plantas daninhas por família e espécie, coletadas em área de rotação arroz irrigado/ melancia há mais de dois anos. Formoso do AraguaiaTO - 2000

\begin{tabular}{|l|l|l|}
\hline \multirow{2}{*}{ Família } & \multicolumn{2}{c|}{ Espécie } \\
\cline { 2 - 3 } & \multicolumn{1}{|c|}{ Nome científico } & \multicolumn{1}{c|}{ Nome comum } \\
\hline \multirow{2}{*}{ Euphorbiaceae } & Chamaesyce hyssopifolia & Erva-de-andorinha \\
\cline { 2 - 3 } & Chamaesyce próstata & Quebra-pedra-rasteira...... \\
\hline \multirow{2}{*}{ Lamiaceae } & Hyptis crenata & Cheirosa \\
\cline { 2 - 3 } & Hyptis lophanta & Cheirosa-de-espiga.................. \\
\hline Compositae & Eclipta alba & Erva-de-botão \\
\hline Onagraceae & Ludwigia sp. & Cruz-de-malta \\
\hline Poaceae & Setaria geniculata & Capim-rabo-de-raposa \\
\hline Solanaceae & Physalis angulata & Balãozinho \\
\hline
\end{tabular}

mecanismos de ação. Contudo, a realização precisa de manejos, como a dessecação, e o uso de pré-emergentes antecedendo o plantio da cultura sucedânea podem reduzir o banco de sementes das plantas daninhas na área, bem como o fluxo de emergência destas durante o ciclo da cultura plantada. Outra alternativa é a manutenção da lâmina de água em profundidades maiores que $15 \mathrm{~cm}$, o que promove controle de gramineas, de plantas de folha larga e de ciperáceas na cultura do arroz irrigado (Barros \& Porto, 1979).

$\mathrm{Na}$ área rotacionada com melancia predominaram as espécies dicotiledôneas, fato

Tabela 6 - Valores de freqüência (F), freqüência relativa (Fr), densidade (D), densidade relativa (Dr), abundância (A), abundância relativa (Ar) e importância relativa (Ir), numa comunidade de plantas daninhas presente em áreas de produção de arroz com rotação de culturas (arroz/melancia) há mais de dois anos. Formoso do Araguaia-TO - 2000

\begin{tabular}{|c|c|c|c|c|c|c|c|c|c|}
\hline Espécie & $\begin{array}{c}\text { Quadro } \\
\text { ocupado }\end{array}$ & $\begin{array}{l}\text { Número de } \\
\text { indivíduos }\end{array}$ & $\mathrm{F}$ & $\begin{array}{l}\mathrm{Fr} \\
(\%)\end{array}$ & $\begin{array}{c}\mathrm{D} \\
\left(\mathrm{pl} \mathrm{\textrm {m } ^ { - 2 }}\right)\end{array}$ & $\begin{array}{l}\mathrm{Dr} \\
(\%)\end{array}$ & A & $\begin{array}{l}\mathrm{Ar} \\
(\%)\end{array}$ & $\begin{array}{l}\mathrm{Ir} \\
(\%)\end{array}$ \\
\hline Physalis angulata & 10 & 703 & 1,00 & 20,00 & 70,30 & 72,70 & 70,30 & 60,41 & 153,10 \\
\hline Eclipta alba & 10 & 108 & 1,00 & 20,00 & 10,80 & 11,20 & 10,80 & 9,28 & 40,50 \\
\hline Hyptis lophanta & 4 & 93 & 0,40 & 8,00 & 9,30 & 9,26 & 23,30 & 19,98 & 37,60 \\
\hline Hyptis crenata & 7 & 24 & 0,70 & 14,00 & 2,40 & 2,48 & 3,43 & 2,95 & 19,43 \\
\hline Chamaesyce prostata & 10 & 22 & 1,00 & 20,00 & 2,20 & 2,28 & 2,20 & 1,89 & 24,17 \\
\hline Ludwigia sp. & 2 & 8 & 0,20 & 4,00 & 0,80 & 0,83 & 4,00 & 3,44 & 8,26 \\
\hline Chamaesyce hyssopifolia & 5 & 7 & 0,50 & 10,00 & 0,70 & 0,72 & 1,40 & 1,20 & 11,93 \\
\hline Setaria geniculata & 2 & 2 & 0,20 & 4,00 & 0,20 & 0,21 & 1,00 & 0,86 & 5,07 \\
\hline Total & - & 967 & 5,00 & 100,00 & 96,70 & 100,00 & 116,00 & 100,00 & 300,06 \\
\hline
\end{tabular}


explicado pela dificuldade de controlar espécies daninhas dicotiledôneas e também monocotiledôneas, em razão da ausência de herbicidas registrados no País para cultura da melancia (Rodrigues \& Almeida, 1998). Considerando que a melancia é cultivada em extensas áreas na região de Formoso do Araguaia-TO, o custo da capina torna-se elevado e ineficiente. Existem na literatura poucos trabalhos que fazem referências à seletividade e ao controle de plantas daninhas com o uso de herbicidas na cultura da melancia. Vidal et al. (2000) verificaram, na melancia Cirullus lanatus cv. Chaleston Gray, alta tolerância ao herbicida fluazifop-p-butil. Já Maciel et al. (2002), avaliando os herbicidas clethodim e sethoxydim, constataram que eles foram altamente seletivos à cultura da melancia, não interferindo na produção e qualidade dos frutos.

Os herbicidas bentazon, bispyribacsodium, clomazone, 2,4-D, ethoxysulfuron, fenoxaprop-ethyl, fenoxaprop-p-ethyl, metsulfuron-methyl, oxadiazon, oxyfluorfen, pendimethalin, propanil e quinclorac são os mais usados pelas cooperativas para o arroz irrigado. Para a soja, os herbicidas acifluorfensódio, acifluorfen+bentazon, clethodim, clomazone, dimethenamid, haloxyfopmethyl, imazaquin, imazaquin+trifluralin, imazethapyr, lactofen, quizalofop-p-ethyl, sethoxydim e trifluralin são os mais utilizados.

Desses herbicidas, bentazon e ethoxysulfuron são indicados no controle das ciperáceas Cyperus esculentus, Cyperus iria e Fimbristylis miliacea, enquanto fenoxaprop-ethyl, fenoxaprop-p-ethyl, imazaquin+trifluralin, oxadiazon, propanil, sethoxydim e trifluralin são eficientes no controle das gramineas Brachiaria plantaginea, Digitaria ciliaris, Digitaria horizontalis, Echinochloa colonum, Eleusine indica e Setaria geniculata (Cruz \& Leiderman, 1978; Santos \& Cruz, 1979; Rodrigues \& Almeida, 1998; Santos et al., 2000). As formulações de trifluralin GrDA $\left(500 \mathrm{~g} \mathrm{~L}^{-1}\right)$ e CE $\left(445 \mathrm{~g} \mathrm{~L}^{-1}\right)$ apresentaram controle eficiente de Brachiaria plantaginea (89 e 94\%), nas doses de 750 e $667 \mathrm{~g} \mathrm{ha}^{-1}$, respectivamente, em trabalho realizado por Laca-Buendia \& Ferreira (2001) na cultura da soja. Fleck et al. (1997), trabalhando com a mesma planta daninha citada anteriormente, obtiveram controle de 93 e 99\% utilizando o herbicida clethodim nas doses de 60 e $120 \mathrm{~g} \mathrm{ha}^{-1}$, respectivamente, aplicado aos 28 dias após a emergência da soja.

Pereira \& Carmona (2000), avaliando o herbicida flumioxazin no controle de Commelina benghalensis na cultura da soja, constataram eficácia de 95 e 96\% nas doses de 20 e $40 \mathrm{~g} \mathrm{ha}^{-1}$, respectivamente; nenhuma toxicidade à soja foi detectada. No entanto, o controle de $99 \%$ da espécie Commelina benghalensis na cultura da soja foi obtido com a mistura dos herbicidas diclosulan+ metolachlor $\left(25+1.200 \mathrm{~g} \mathrm{ha}^{-1}\right)$ aos 42 dias após a aplicação (Oliveira Jr. et al., 2002). Os herbicidas 2,4-D, imazethapyr e lactofen controlam as folhas largas Ageratum conyzoides, Commelina benghalensis, Eclipta alba, Hyptis lophanta, Ipomoea grandifolia, Physalis angulata e Portulaca oleracea (Rodrigues \& Almeida, 1998); o 2,4-D também controla as espécies Chamaesyce hirta, Chamaesyce hyssopifolia, Emilia sonchifolia, Ludwigia sp. e Senna obtusifolia.

Em se tratando do controle das espécies Chamaesyce prostata, Hyptis crenata, Malachra fasciata e Murdannia nudiflora, não há herbicidas registrados para nenhuma das culturas estudadas (Rodrigues \& Almeida, 1998).

Quando são comparadas as três áreas de produção de arroz irrigado, nas quais foram empregados manejos culturais distintos, podese observar que as comunidades de plantas daninhas apresentaram modificações na sua composição florística de acordo com o tipo de manejo utilizado. Dessa forma, com base nos resultados, verificou-se que, na área de produção de arroz irrigado sem rotação de cultura há mais de cinco anos, houve maior predomínio das espécies das famílias Poaceae e Compositae; na área rotacionada com a cultura da soja há mais de cinco anos o maior predominio foi das espécies das famílias Poaceae e Cyperaceae, enquanto na área rotacionada com a cultura da melancia há mais de dois anos as familias Euphorbiaceae e Lamiaceae apresentaram o maior número de espécies.

A utilização de um mesmo sistema de cultivo em uma determinada área por vários anos consecutivos pode aumentar a pressão 
de seleção sob as comunidades de plantas daninhas, selecionando as espécies mais adaptadas ao sistema de cultivo, bem como favorecer o surgimento de plantas resistentes a herbicidas.

\section{LITERATURA CITADA}

BARROS, L. C. G.; PORTO, E. R. Efeito da profundidade da lâmina de água sobre a incidência de plantas na cultura do arroz. Planta Daninha, v. 2, n. 2, p. 69-72, 1979.

BRAUN-BLANQUET, J. Fitossociologia: bases para el estudio de las comunidades vegetales. Madrid: H. Blume, 1979. 820 p.

CRUZ, L. S. P.; LEIDERMAN, L. Controle de plantas daninhas em cultura de soja (Glycine $\max$ L. Merril) com misturas de herbicidas. Planta Daninha, v. 1, n. 1, p. 1317,1978 .

CURTIS, J. T.; MCINTOSH, R. P. The interrelations of certain analytic and synthetic phytossociological characters. Ecology, v. 31, p. 434-455, 1950.

FLECK, N. G.; CUNHA, M. M.; VARGAS, L. Dose reduzida de clethodim no controle de papua na cultura da soja, em função da época de aplicação. Planta Daninha, v. 15, n. 1 , p. 18-24, 1997.

JAPAN INTERNATIONAL COOPERATION AGENCY JICA. The integrat development master plan study agriculture and livestock of the state of Tocantins. Japan: JICA, annex, v. 2, p. 1-4, annex 4, 1998.

LACA-BUENDIA, J. P.; FERREIRA, J. C. Eficácia agronômica de formulações e doses de trifluralin na cultura da soja. R. Bras. Herb., v. 2, n. 1, p. 43-47, 2001.

MACIEL, C. D. G.; CONSTANTIM, J.; GOTO, R.

Seletividade e eficiência agronômica de herbicidas no controle de capim-colchão na cultura da melancia. Hortic. Bras., v. 20, n. 3, p. 474-476, 2002.
MARTINS, F. R. Critérios para a avaliação de recursos naturais. In: SIMPÓSIO SOBRE A COMUNIDADE VEGETAL COMO UNIDADE BIOLÓGICA, TURÍSTICA E ECONÔMICA, 1978, São Paulo. Anais... São Paulo: Academia de Ciências do Estado de São Paulo, 1978. p. 136-149. (Publicação ACIESP, 15).

MARTINS, F. R. Esboço histórico da fitossociologia florestal no Brasil. In: CONGRESSO BRASILEIRO DE BOTÂNICA, 1985, Curitiba. Anais... Curitiba: IBAMA, 1985. p. 33-60.

MUELLER-DOMBOIS, D.; ELLENBERG, H. Aims and methods of vegetation ecology. New York: John Wiley \& Sons, 1974. 547 p.

OLIVEIRA Jr., R. S. et al. Controle de plantas daninhas e seletividade de diclosulam aplicado em pré-emergência na cultura da soja. R. Bras. Herb., v. 3, n. 1, p. 69-74, 2002.

PEREIRA, R. C.; CARMONA, R. Eficácia do herbicida flumioxazin, isolado e em mistura com sulfosate, no manejo de plantas daninhas em plantio direto de soja. Revista Brasileira de Herbicidas, v. 1, n. 2, p. 107-112, 2000.

RODRIGUES, B. N.; ALMEIDA, F. S. Guia de herbicidas. 4.ed. Londrina, 1998. $648 \mathrm{p}$.

SANTOS, C. A. L.; CRUZ, L. S. P. Efeito do bentazon e Bentazon+Dichlorprop na cultura do arroz irrigado e sobre as plantas daninhas. Planta Daninha, v. 2, n. 1, p. 18-21, 1979.

SANTOS, F. J. et al. Controle químico de plantas daninhas na cultura do arroz irrigado no Estado do Ceará. Planta Daninha, v. 18, n. 1, p. 29-37, 2000.

SORENSEN, T. A. Method of stablishing groups equal amplitude in plant society based on similarity of species content. In: ODUM, E. P. Ecologia. 3.ed. México: Interamericana, 1972. p. 341-405.

VIDAL, R. A. et al. Seletividade do herbicida fluazifop-pbutil para cucurbitáceas. Planta Daninha, v. 18, n. 3, p. 413-417, 2000. 